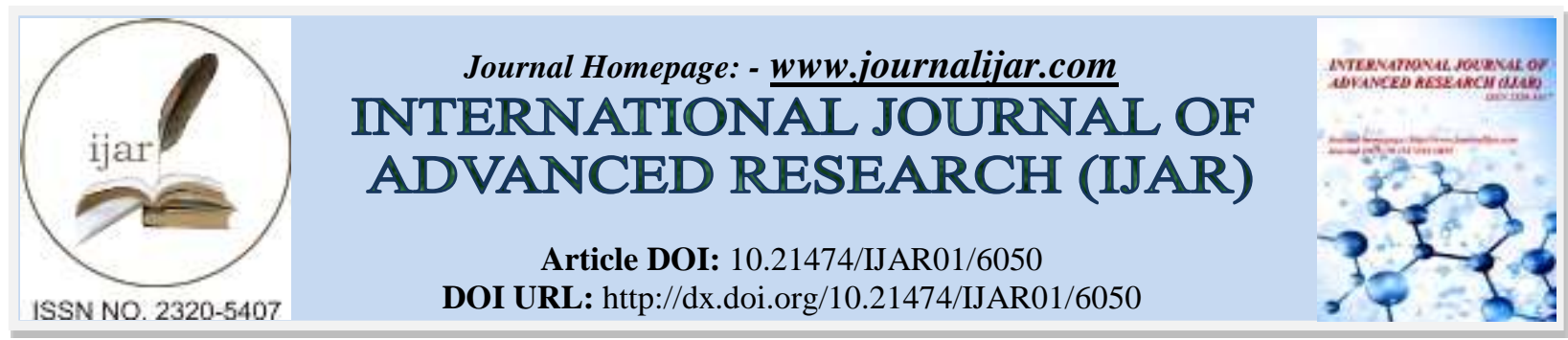

RESEARCH ARTICLE

\title{
DOSE THE USE OF VISUAL BIOFEEDBACK IN ELDERLY BALANCE TRAINING MINIMISE THEIR INCIDENCE OF FALLING?
}

\author{
Abdullah Abbas Tobaigy, M. Sc. ${ }^{1}$ and Mansour Abdullah Alshehri, M. Sc. ${ }^{2}$ \\ 1. College of Medicine and Health, University College Cork, Ireland. \\ 2. Physiotherapy Department, Faculty of Applied Medical Sciences, Umm Al-Qura University, Saudi Arabia.
}

\section{Manuscript Info}

Manuscript History

Received: 13 October 2017

Final Accepted: 15 November 2017

Published: December 2017

Key words:-

Visual biofeedback, Balance, Falling,

Risk of falling, Elderly, Geriatric.

\section{Abstract}

Falling is an issue for people of all ages, not just the elderly, resulting in injuries including head traumas and bone fracture. Balance impairment is one of the most reported issues to physicians and experienced by older people. Visual biofeedback (VBF) is a type of rehabilitation treatment tool that provides an individual with visual information about their centre of gravity within the limits of stability, while they stand on a pressure plate. This report aimed to critically evaluate studies that used the VBF intervention with elderly people in term of balance improvement and reducing the risk of falling. Various studies used different types of measurement to assess falling and the risk of falling. Despite the limitations of the studies, the results from the studies demonstrated a positive trend in improving the balance of older people and decreasing the risk of falling. In conclusion, it is evident that VBF significantly improved the balance of the elderly, so further research should be conducted to investigate the type, frequency and duration of VBF and assess the short and long-term effects of using VBF intervention in balance to reduce the incidence of falling.

Copy Right, IJAR, 2017,. All rights reserved.

\section{Introduction:-}

Falling is an issue for people of all ages, not just the elderly, resulting in injuries including head traumas and bone fracture. Approximately 30 to $35 \%$ of elderly people in community experience at least one fall a year (Gusi et al., 2012 ) and the treatment of such falls is costly. Indeed, according to the National Institute for Clinical Excellence (NICE), the annual cost of falling in the United Kingdom is estimated to be $£ 2.3$ billion (NICE, 2013).

Balance impairment is one of the most reported issues to physicians and experienced by older people (Barin \& Dodson, 2011). According to Lin and Bhattacharyya (2012), one in five elderly people in USA have experienced complications with balance and dizziness per annum. Balance is defined as the ability of human body to keep their centre of gravity within the base of support (Walker, Brouwer \& Culham, 2000). The effect of performing exercise to decrease falling or the risk of falling and improve the balance have been well documented in some clinical practice guidelines for fall prevention such AGS/BGS (2010) and NICE (2013). 
Visual biofeedback (VBF) is a type of rehabilitation treatment tool that provides an individual with visual information about their centre of gravity within the limits of stability, while they stand on a pressure plate. The person can see where their centre of gravity is relative to the support base shown on a screen (Walker, Brouwer $\&$ Culham, 2000). Simply, the individual stands above a force plate in front of screen that allows them to observe their performance while performing the task. Nowadays, there are many types of VBF used such as the Nintendo Wii Fit.

\section{Discussion:-}

Sihvonen, Sipilä and Era (2004) published a study (conducted between 2002 and 2003) about the change in postural balance in 27 frail elderly women from two residential care facilities. There were 20 participants in the experimental group (EG), with a mean age of $80.7 \pm 6.1$ years and 7 participants in the control group (CG) with a mean age of $82.9 \pm 4.2$ years. The EG performed VBF training three times a week for four weeks. The functional balance of the participants was measured by the dynamic balance test, standing balance test and the Berg Balance Scale. The EG had statistically significant improvement in the dynamic balance test by $35.9 \%$ and performance distance by $28.2 \%$ (where the baseline data was mentioned in the article) in comparison to the CG, which increased $0.6 \%$ on the time and $9.8 \%$ on the distance. Also, the berg balance scale of the EG increased by $6.9 \%$, whereas the CG improved by only $0.7 \%$. Finally, with regards to standing balance, there was no significant change from baseline, but the EG group scored better at the highest difficulty level. The authors concluded that their result showed an improvement in the balance of elderly frail women using VBF training who lived in care facilities. They recommended that further research was performed in frail elderly populations with different medical conditions.

Subsequently, Sihvonen et al. conducted a further study about fall incidence after visual feedback balance training for frail elderly women. They followed up their 27 participants for one year after finishing their intervention in 2003. They interviewed them about their physical activity level, medications that they used and incidence of falling last year. Also, they assessed their fear of falling level by asking one closed question, the answers to which were no, yes: some and yes: a lot. The same question was asked at follow up in 2004. Participants were asked to complete daily diaries and send it to them by email at the end of every month for a year. The researchers contacted individual participants by phone if they did not return their diary, sent incomplete diaries or reported a falling incidence. The results showed that 5 out of 7 of the CG reported falling, with 3 suffering from recurrent falling. The total falling incidence was 11 times for all. In the EG, 11 experienced falling, 1 participant fell twice, with a total of 12 falls. Also, they found the level of fear of falling declined and the level of physical activity increased in EG group after the intervention. The level of changes decreased after the one year follow up, with no significant change detected in the CG.

The methodology in both studies was very clear and well explained. The participants were randomised to the study group by drawing lots, with an unequal number in each group. The sample size for both groups was small, which may have affected the external validity of the study. In addition, they did not mention how and why they chose these two residential care facilities in the first and second study, or why the number in each group was not equal, which may have resulted in selection bias in favour of the experimental group. Finally, they did not clarify whether the study was blinded.

Gusi et al. (2012) conducted a controlled randomised trial to determine whether balance training could reduce the fear of falling, as well as improving dynamic balance and isometric strength in the elderly. This study was better than Sihvonen, Sipilä and Era (2003) in many aspects, even though the study design was different. First, they had a larger sample size of forty institutionalised older people divided equally between the groups, EG and CG. The participants were randomised into groups and it was triple blinded trial. The mean age for both groups was $76 \pm 8$ years. They used the Biodex Balance System (BBS) platform for balance training and measuring dynamic balance and isometric strength in their experimental group. Both groups were required to complete an international questionnaire, the Falls Efficacy Scale, which was developed by the Prevention of Falls Network Europe, to measure their fear of falling. Also, this questionnaire is a widely accepted tool to measure the fear of falling due to its very high validity and reliability (Yardley et al., 2005).

Multidisciplinary care, like nursing, physiotherapy and occupational therapy, was given to both groups, with the EG receiving extra training via the $\mathrm{BBS}$, which focused on weight shift, balance and rebalance exercises. In addition, the BBS can measure the dynamic balance by performing the Fall Risk Test (BMS, 1999 cited in Gusi et al., 2012). The duration of the exercise was 15 minutes, with two sessions per week for twelve weeks. The results showed that there were improvements in EG with regard to their fear of falling decreasing by 7 points (SD 7), whereas it only 
decreased by 1 point (SD 4) in the CG. For dynamic balance, the EG improved more than the CG group, finishing the intervention by 2.1 (95\% CI 1.3 to 3.0). Furthermore, there is a significant increase in the knee isometric strength (knee flexor and extensor muscles) for EG group compared to CG, who showed a slight decrease in their strength. They explained that their results were due to an improvement in the knee isometric extensor muscles strength leading to an improved dynamic balance by $29 \%$. This increase in dynamic balance resulted in a decrease in the fear of falling among the participants. In this study, the methodology used and the study results were clearly presented. Both the studies by Sihvonen, Sipilä and Era (2003) and Gusi et al. (2012) performed a dynamic balance exercise and test to improve the balance in older people and decrease the fear and risk of falling. Their results were in agreement, that improvement in dynamic balance could lead to a reduced risk of falling. However, the study of Gusi et al. (2012) was limited as they stated "The clinical trial registration did not specify a single primary outcome so the Falls Efficacy Scale was nominated post hoc" (Gusi et al., 2012). In addition, their results cannot be generalised as their participants were institutionalised elderly people, not elderly people living in the community.

As the technology of VBF continues to improve, their application in rehabilitation has increased, with many researchers using exergames or video games as intervention. According to Lai et al. (2013), simply performing exercise via games creates an interactive environment to do exercise, which is more attractive and feasible to healthy elderly than normal or standard exercise. So, Lai et al. conducted a prospective crossover, single blinded randomised trial to investigate the effect of interactive video game based (IVGB) systems on elderly balance exercise. The study involved 30 community-dwelling participants aged over 65 (mean age 72.1 years, SD 4.8) randomly allocated equally in two groups, A and B. Participants were excluded if they suffered from cardiovascular disease that could affect their walking ability, neurological diseases like stroke, visual impairment, walking with assistance and having an arthritis. The Xavix measured step system connected to a television and laptop was used as IVGB intervention for 12 weeks of exercise, 30 minutes per session, three times per week. Group A underwent the IVGB intervention for the first 6 weeks, then changed to the control phase for the following 6 weeks, whereas group B started the intervention in the control phase for the first six weeks, then became the experimental group for the next six weeks.

The outcomes were measured by the Berg Balance Scale (BBS) for functional balance, timed up and go for functional mobility for elderly. The Modified Falls Efficacy Scale (MFES) was used to assess how much confidence older people have that they are not going to fall during doing their daily life activities and "it has demonstrated strong retest reliability" (Hill et al., 1996). The Unstable Surface Training (UST) was used to for assisting static balance and "it is a valid measure for balance and predicts injurious falls" (Lai et al., 2013). Also, Sway Velocity (SV) and Sway Area (SA) were used as an outcome measurement, both of which can be deducted by The Catsys 2000 system which tests postural sway. The final measurement was the XMSS stepping test, in which the participant has to stand on a platform with a sensory detector facing screen and then, step on the corresponding platform trying to mimic the movements that appear on the screen. There was a significant increase in BBS, MFES, SV with both open and close eyes, TUG and XMSS stepping test for both legs in group A after the first 6 weeks IVGB intervention, but no significant difference in SA and UST. In addition, after the next 6-week control phase, group A results were not significantly different from the 6-week results, suggesting that the effect of training remained even after the control phase without intervention. For group B, there was no significant difference in measurements after the control phase. However, after the 6 weeks of IVGB intervention, there were significant improvements in all measurements, except UST which was not significant. According to the study findings, the researchers assumed that the intensity and duration used in their intervention may have a sufficient effect on both groups.

Nonetheless, this study had some limitations. First, they did not mention how they randomised the participants and examiners were not blinded to the participants, which may lead to information bias. Also, the Xavix system has not been commonly or widely used. In addition, they did not measure the fall statues for participants but they assessed the participant's balance objectively by measurements that have been demonstrated clinically to make predictions about falls. However, the discussion was very clear. The results showed a significant improvement in BBS in this study as well as the study by Sihvonen, Sipilä and Era (2004). According to Shumway-Cook et al. (1997), BBS is the highest single accurate predictor for falling and state that "in the range of 54 to 46, a 1-point change in the Berg Balance Scale scores led to a $6 \%$ to $8 \%$ increase in fall risk" (Shumway-Cook et al., 1997).

\section{Summary:-}

Overall, the different types of visual biofeedback used in the aforementioned studies were used to assess various important elements in balance that could improve balance in elderly, such as dynamic and static balance and postural sway. Also, the various studies used different types of measurement to assess falling and the risk of falling. 
The results from the studies demonstrated a positive trend in improving the balance of older people and decreasing the risk of falling. Despite the limitations of the studies, visual biofeedback in training the elderly is effective, as explained by Kang (2013), sensory integration produces righting reflex and trunk reactions through visual feedback. These studies further support the hypothesis that VBF training can improve balance. Also, numerous studies involved using VBF in training in some neurological conditions. Zijlstra et al. (2010) mentioned in their systematic review that two out of three studies which investigated $\mathrm{VBF}$ intervention for post stroke patients to train their balance, found a significant improvement toward the distribution of patients' weight while standing. In general, the studies discussed in the present review has small sized population samples and they investigated specific aspects of balance in healthy and frail old people. There was agreement between the studies of Sihvonen, Sipilä and Era (2004) and Gusi et al. (2012) in favour of improved dynamic balance, as well as Lai et al. (2013) and Sihvonen, Sipilä and Era (2004) with regard to the BBS results.

However, these systems, such as the Biodex Balance system, that were used for assessment and training dynamic balance (Aydog et al., 2006) are costly (€1200) and may not be affordable for all clinicians (Gusi et al., 2012). In comparison, exergames are relatively inexpensive and easy to access; VBF could be performed using the Nintendo Wii Fit for example. Also, older people seem to enjoy and accept exercising using exergames. According to pilot study conducted by Agmon et al. (2011), seven older people reported enjoying exercising with their grandsons using the Nintendo Wii Fit.

Only one study (Sihvonen et al., 2004) followed 27 participants for one year, which is considered to be a good point at which to detect the long-term risk of falling. For other studies (Gusi et al., 2012), participants were not followed after finishing the intervention, with measurements taken immediately after the 12-week intervention. Also, for Lai et al. (2013), the A group was only followed for 1 week to determine an effect from their intervention. Zijlstra et al. (2010) concluded that there is still a need for further studies to assess the long-term effects of interventions to be able to determine the different functional rehabilitation needs of the elderly.

\section{Conclusion:-}

In conclusion, it is evident that VBF significantly improved the balance of the elderly, so further research should be conducted to investigate the type, frequency and duration of VBF and assess the short and long-term effects of using VBF intervention in balance to reduce the incidence of falling, as well as the fear of falling.

\section{References:-}

1. Agmon, M., Perry, C., Phelan, E., Demiris, G. and Nguyen, H. (2011): A pilot study of Wii Fit exergames to improve balance in older adults. J. Geriatr. Phys. Ther., 34(4): 161-167.

2. American Geriatrics Society. (2010): AGS/BGS clinical practice guideline for the prevention of falls in older persons.

[online]. www.americangeriatrics.org/health_care_professionals/clinical_practice/clinical_guidelines_recommendations/ 2010/ (Accessed 15 November 2016).

3. Aydog, E., Bal, A., Aydog, S.T. and Cakci, A. (2006): Evaluation of dynamic postural balance using the Biodex Stability System in rheumatoid arthritis patients. Clin. Rheumatol., 25(4): 462-467.

4. Barin, K. and Dodson, E. (2011): Dizziness in the elderly. Otolaryngol Clin. North. Am., 44(2): $437-454$.

5. Gusi, N., Carmelo Adsuar, J., Corzo, H., Del Pozo-Cruz, B., Olivares, P.R. and Parraca, J.A. (2012): Balance training reduces fear of falling and improves dynamic balance and isometric strength in institutionalised older people: a randomised trial. J Physiother., 58(2): 97-104.

6. Hill, K.D., Schwarz, J.A., Kalogeropoulos, A.J. and Gibson, S.J. (1996): Fear of falling revisited. Arch. Phys. Med. Rehabil., 77(10): 1025-1029.

7. Kwon, K. (2013): Effects of Visual Biofeedback Training for Fall Prevention in the Elderly. J. Phys. Ther. Sci., 25(11): 1393-1395.

8. Lai, C.H., Peng, C.W., Chen, Y.L., Huang, C.P., Hsiao, Y.L. and Chen, S.C. (2013): Effects of interactive video-game based system exercise on the balance of the elderly. Gait. Posture., 37(4): 511-515.

9. Lin, H. and Bhattacharyya, N. (2012): Balance disorders in the elderly: epidemiology and functional impact. Laryngoscope., 122(8): 1858-1861.

10. National Institute for Clinical Excellence. (2013): Falls: The Assessment and Prevention of Falls in Older People. [online]. www.nice.org.uk/guidance/CG161 (Accessed 25 November 2016). 
11. Shumway-Cook, A., Baldwin, M., Polissar, N.L. and Gruber, W. (1997): Predicting the probability for falls in community-dwelling older adults. Phys Ther., 77(8): 812-819.

12. Sihvonen, S. Sipilä, S. and Era, P. (2004): Changes in postural balance in frail elderly women during a 4-week visual feedback training: a randomized controlled trial. Gerontology., 50(2): 87-95.

13. Sihvonen, S., Sipilä, S., Taskinen, S. and Era, P. (2004): Fall incidence in frail older women after individualized visual feedback-based balance training. Gerontology., 50(6): 411-416.

14. Walker, C., Brouwer, B.J. and Culham, E.G. (2000): Use of visual feedback in retraining balance following acute stroke. Phys Ther., 80(9): 886-895.

15. Yardley, L., Beyer, N., Hauer, K., Kempen, G., Piot-Ziegler, C. and Todd, C. (2005): Development and initial validation of the Falls Efficacy Scale-International (FES-I). Age Ageing., 34(6): 614-619.

16. Zijlstra, A., Mancini, M., Chiari, L. and Zijlstra, W. (2010): Biofeedback for training balance and mobility tasks in older populations: a systematic review. J Neuroeng Rehabil., 7(1): 1. 